\title{
Topical anaesthesia with local anaesthetic (lidocaine and prilocaine, EMLA) cream for cautery of genital warts
}

\author{
A HALLÉN, K LJUNGHALL, J WALLIN \\ From the Department of Dermatology and Venereology, University Hospital, Uppsala, Sweden
}

SUMMARY Removal of genital warts by thermocautery was performed in 108 patients (57 men and 51 women) under topical anaesthesia with a local anaesthetic cream, lidocaine and prilocaine (EMLA). Most men had warts in the preputial cavity, most women had warts situated on the mucous membranes of the vulva, and warts at multiple sites were common. About $1 \mathrm{ml}$ of cream per lesion was applied to the warts for 20 to 105 minutes before the operation. Plastic film (Glad, Union Carbide) was applied over the cream when natural occlusion, such as under the prepuce or on the introitus, was not present. Local pallor was seen in $30 \%$ of the patients, redness in $53 \%$, and oedema in $15 \%$, but did not cause any discomfort and were clinically insignificant. Analgesia was sufficient in $96 \%$ of the men and in $40 \%$ of the women. Additional local infiltration was given to $60 \%$ of the women, but was not as painful as injections generally are in the genital area. The analgesic efficacy on women may be further improved by optimising the application time on the genital mucosa.

The reported number of patients with genital warts (condylomata acuminata) is increasing, and the disease is currently three times as common as genital herpes. ${ }^{1}$ It is classified as a sexually transmitted disease (STD). Condylomata are caused by human papillomavirus (HPV), which is probably associated with vulval and cervical cancer. ${ }^{2}$ Treatment is therefore important to avoid malignant transformation and to decrease the risk of further transmission. Local application of cytotoxic agents, such as podophyllotoxin, is recommended as initial treatment. ${ }^{3}$ Remaining warts are usually removed surgically by cautery, diathermy, laser, or excision. Intraanal and intraurethral condylomata, as well as warts located in the vagina or on the cervix, are preferably removed surgically without pretreatment with podophyllotoxin.

Cautery is painful and requires analgesia, which may be provided by local infiltration anaesthesia. The genital area is very sensitive, however, and injections are painful in most cases. Treatment of larger areas

Address for reprints: Dr Anders Hallén, Department of Dermatology and Venereology, University Hospital, S-751 85 Uppsala, Sweden.

Accepted for publication 12 March 1987 may necessitate general anaesthesia.

A new local anaesthetic cream containing two well known local anaesthetics, lidocaine and prilocaine, offers analgesia without the use of needles. The cream, EMLA (eutectic mixture of local anaesthetics, Astra Alab AB), provides sufficiently good anaesthesia for an intravenous cannula to be inserted about one hour after applying the cream to the skin and covering it with an occlusive dressing. ${ }^{4}$ When it is applied to mucocutaneous areas, absorption is likely to be more rapid.

A pilot study indicated that the cream could be useful as analgesia for the removal of genital warts. ${ }^{5}$ The present open trial was performed to evaluate the use of lidocaine and prilocaine cream as a local anaesthetic for cautery or surgical excision of condylomata acuminata.

\section{Patients and methods}

\section{PATIENTS}

One hundred and eight outpatients (57 men and 51 women) attending the STD department of the University Hospital, Uppsala, with genital warts were included in the trial. Patients who were pregnant or 
had a known allergy to local anaesthetics were not allowed to participate. Concomitant medication with analgesics was not allowed. Before admission to the trial, the patients were given written and verbal information on the purpose of the investigation, in accordance with the Helsinki Declaration, and gave their verbal consent to participate. The men were aged 17 to 59 (median 25) and the women 16 to 41 (median 21).

Of the 57 men, 51 had warts in the preputial cavity, seven in the urinary meatus, 10 on the penile shaft, and two on the scrotum. Like the men, most women had warts in more than one location. In 44 women warts were located on the mucous membranes of the vulva, and in 34 there were warts on the skin, most often on the perineum or the perianal area.

\section{DRUG, DOSAGE, AND APPLICATION TIME}

EMLA cream containing lidocaine $25 \mathrm{mg}$ and prilocaine $25 \mathrm{mg}$ was used. About $1 \mathrm{ml}$ of cream was applied to each area with lesions, up to a maximum of $10 \mathrm{ml}$ per patient. The intended application time of the cream was at least 30 minutes for mucous membranes and 60 minutes for normal skin. The estimated doses and sizes of the treated areas and the application times in men and women are listed in the table. The dose was estimated roughly $(1 \mathrm{ml}$ being about one fifth of a tube).

Plastic film (Glad, Union Carbide) was applied over the cream when natural occlusion, such as under
Table Local anaesthesia of condylomata with lidocaine and prilocaine (EMLA) cream. Mean (range) of areas treated, doses used, and application times in men and women

\begin{tabular}{llll}
\hline & $\begin{array}{l}\text { Treated area } \\
(\text { sq mm })\end{array}$ & $\begin{array}{l}\text { Dose } \\
(\mathrm{ml})\end{array}$ & $\begin{array}{l}\text { Application } \\
\text { time }(\text { mins })\end{array}$ \\
\hline Men $(\mathrm{n}=57)$ & $18(1-1800)$ & $3(1-6)$ & $\begin{array}{l}35(20-70) \\
50(30-105) \dagger\end{array}$ \\
Women $(\mathrm{n}=51)$ & $50(2-1600)^{*}$ & $3(1-8)$ & 50 \\
\hline
\end{tabular}

Data missing for * two women and tone woman.

the prepuce or on the introitus, was not present. Men were allowed to walk around until the time of operation. The cream was applied to women in the lithotomy position. They then remained on a nearby couch until the time of operation.

\section{LOCAL REACTIONS}

After wiping off the cream, the physician examined the anaesthetised area for redness, pallor, or oedema, which was rated as none, slight, moderate, or severe.

\section{SURGER Y}

Thermocautery was used in 56 men and both thermocautery and excision in one. Remnants of the warts were scraped off with a curette at nine of the operations. In the women, thermocautery was used in $\mathbf{4 8}$ patients, and 24 of the operations were completed by curettage. In two women both thermocautery and excision were used. One woman was treated by carbon dioxide laser.

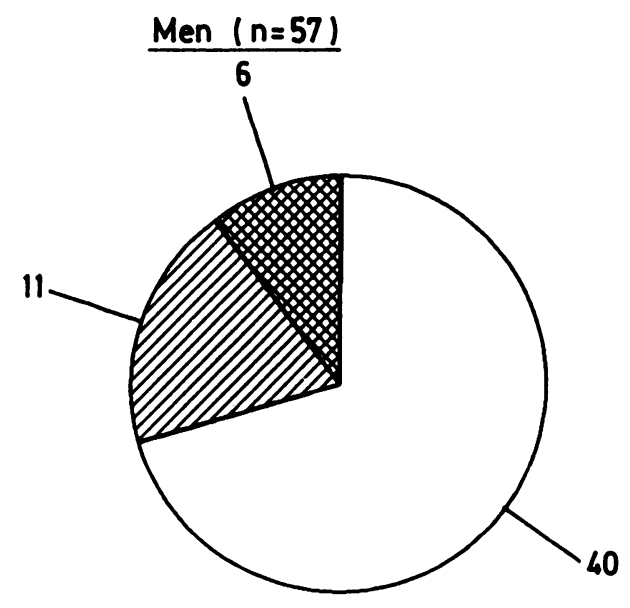

Pain: $\square$ None

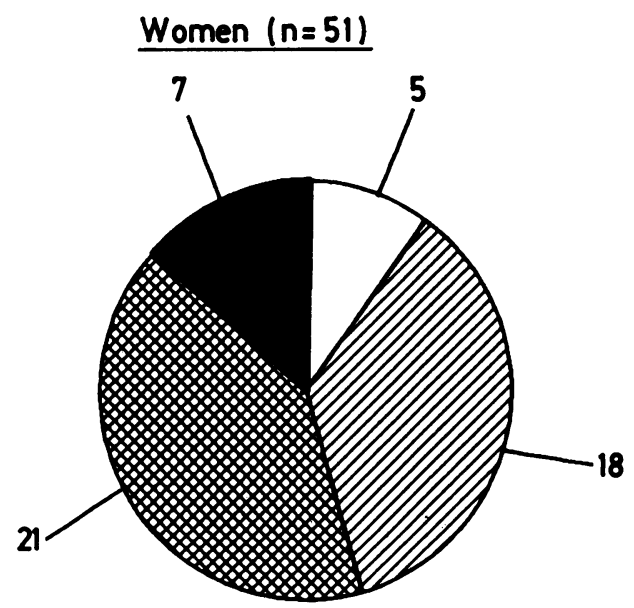

Moderate

Severe

Fig 1 Physicians' pain evaluation of condyloma treatment after lidocaine and prilocaine cream (EMLA) anaesthesia. 
EVALUATION OF PAIN

The pain experienced during the operation was recorded by the operating physician on a four point scale as none, slight, moderate, or severe. When multiple warts were present, the assessment was based on the strongest reaction of the patient. After the operation, the patient was questioned about the overall degree of pain. The result was recorded on a separate form, using a five point scale stating that the pain was: none, mild, neither mild nor severe, rather severe, severe.

If the anaesthesia was unsatisfactory, additional analgesia was provided either by applying more lidocaine and prilocaine cream or by local infiltration of lignocaine hydrochloride $1 \%$. Details of the additional treatment were recorded. In the last part of the trial, 20 women given lignocaine hydrochloride injections were also asked to evaluate the pain caused by the injection. For this purpose, the ratings no, slight, or severe pain were used.

\section{Results}

EVAluations of Pain

Figure 1 shows the physicians' evaluations of pain experienced by the patients. Of the 57 men, $51(90 \%)$ felt no or only slight pain during the treatment, and none felt severe pain. There was no difference in pain during treatment of sessile and hyperplastic warts. No pain was felt by the three patients in whom excision was used. Of the 51 women, $23(45 \%)$ were judged to have felt no or slight pain. Seven women felt severe pain. One woman with multiple warts on the introitus was treated by carbon dioxide laser on an area of $20 \times 30 \mathrm{~mm}$ without any pain. This operation was performed 35 minutes after the cream had been applied.

The patients' pain evaluations are shown in figure 2. For both men and women the patient's grading of the pain agreed with that of the doctor.

Treatment was interrupted and additional analgesia given to four of the six men with moderate pain. Two were given lidocaine hydrochloride and two were given more lidocaine and prilocaine cream. Treatment was continued two and five minutes later. The pain from the continued treatment was rated as slight by the patients given more cream. The cream thus gave sufficient analgesia for cautery of genital warts in 55 of 57 men (97\%).

Additional lidocaine hydrochloride infiltration was given to 31 women. Six had felt slight and 25 moderate or severe pain (physician's evaluation). The pain experienced from the additional injection was recorded separately for the last 20 women; 18 rated

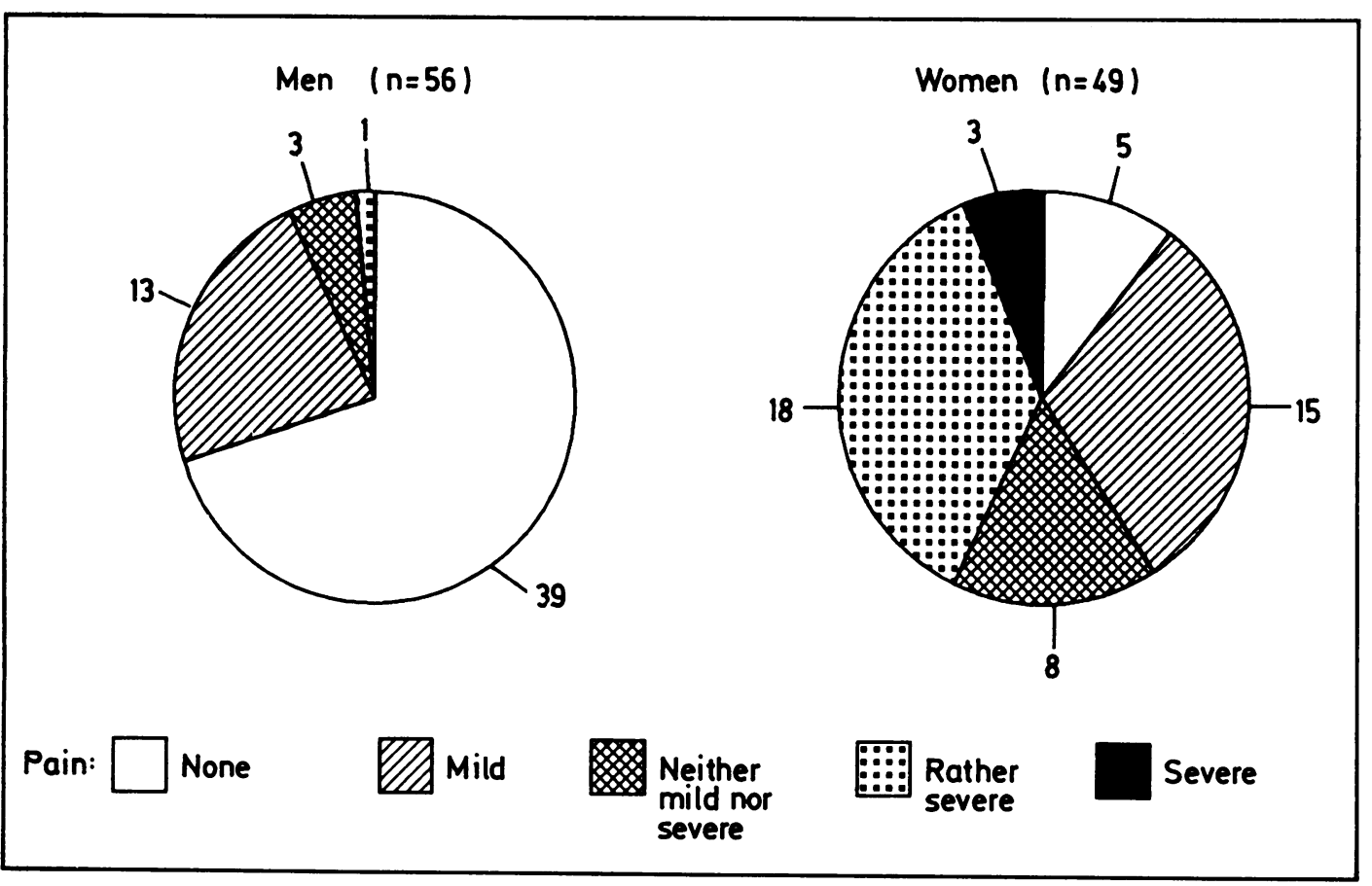

Fig 2 Patients' pain evaluation of condyloma treatment after lidocaine and prilocaine cream (EMLA) anaesthesia (data for one man and two women missing). 
the pain as either none or slight, and in two women it was severe.

\section{LOCAL REACTIONS}

None of the 108 patients spontaneously complained of any symptoms due to the applied cream before the operation. Local reactions were recorded in about $50 \%$ of the patients, most often a slight central erythema with pallor at the outer edges. The erythema in two of the men was rated as severe. One woman had severe and another moderate oedema. These reactions, however, were not considered to be clinically important, required no further treatment, and did not interfere with the intended operation.

\section{Discussion}

Lidocaine and prilocaine cream gave sufficient analgesia for cautery of genital warts in $97 \%$ of the men, a most satisfactory result. An application time of at least 60 minutes was recommended on the skin. In this trial, however, seven men with condylomata on the scrotum or the penile shaft were treated after application times of only 20 to 35 minutes, with no or only slight pain. The thin stratum corneum of scrotal and penile skin may allow faster penetration of the local anaesthetics than other skin.

Almost all injections in the genital area are very painful. Additional analgesia was required in $60 \%$ of the women. Patients who had previously received infiltration anaesthesia of the genitals, however, found that the injection was much less painful after pretreatment with the cream.

There was an obvious difference in efficacy between men and women. The application time in the women (median 50 minutes) was longer than in the men (median 35 minutes). The difference in application time between men and women suggests that the local anaesthetics in the cream were absorbed and eliminated more rapidly than expected. Better analgesia of the genital mucosa can possibly be obtained sooner than of the skin after application of the cream. In an additional open trial, performed during the preparation of this report, nine men with meatal condylomata and 11 men with condylomata in the preputial cavity were treated three to 27 minutes (median 9.5 minutes) after the application of lidocaine and prilocaine cream. Seventeen men experienced no pain and the remaining three only slight pain, which did not interfere with the treatment. A recent investigation of the oral mucosa showed that lidocaine and prilocaine cream reduces the pain from needle insertion only two to five minutes after application. ${ }^{6}$ Thus it seems that shorter application times can be used on mucosal areas.

The duration of mucosal anaesthesia may depend on the dose administered - that is, the thickness of the layer of cream. Improved analgesic efficacy on the genital mucosa in women after similar application times as in this trial may also be achieved by a higher dose of the cream.

The recorded number of local reactions was high, but they did not cause any discomfort for the patients. Earlier studies have shown that the cream causes transient redness of the skin in about $30 \%$ of the patients, ${ }^{78}$ and Ohlsén et al suggested that the redness may be caused by the vasoactive effects of lidocaine and prilocaine. ${ }^{8}$ In cases where oedema was present it actually facilitated the identification of the flat warts.

In conclusion, lidocaine and prilocaine cream proved to be effective as topical anaesthetic for cautery of genital warts in men. In women, it was effective in $40 \%$ of the patients, and a combination of lidocaine and prilocaine cream and infiltration was quite satisfactory in the remainder. The results imply that the analgesic effect in women may be further improved by optimising the application time on the genital mucosa.

We thank Astra Alab AB, Södertälje, Sweden, for supplying the drug and for technical help with the management of this trial.

\section{References}

1 McMillan A. Sexually transmitted diseases: an overview. Practitioner 1985;229:971-7.

2 Singer A, Walker PG, McCance DJ. Genital wart virus infections: nuisance or potentially lethal?. $\mathrm{Br} \mathrm{Med} J$ 1984;288:735-7.

3 von Krogh G. Podophyllotoxin for condylomata acuminata eradication. Acta Derm Venereol (Stockh) 1981:suppl 98.

4 Ehrenström-Reiz GME, Reiz SLA, Stockman O. Topical anaesthesia with EMLA, a new lidocaine-prilocaine cream and the Cusum technique for detection of minimal application time. Acta Anaesthesiol Scand 1983;27:510-2.

5 Juhlin L, Evers H, Broberg F. A lidocaine-prilocaine cream for superficial skin surgery and painful lesions. Acta Derm Venereol (Stockh) 1980;60:544-6.

6 Holst A-L, Evers H. Experimental studies of new topical anaesthetics on the oral mucosa. Swed Dent $J$ 1984;8:295.

7 Dohlwitz A, Uppfeldt A. Schmerzlinderung bei Venenpunktion. Applikationszeit und Wirksamkeit einer Lidocain-PrilocainCreme. Anaesthesist 1985;34:355-8.

8 Ohlsén L, Englesson S, Evers H. An anaesthetic lidocaine/ prilocaine cream (EMLA) for epicutaneous application tested for cutting split skin grafts. Scand J Plast Reconstr Surg 1985;19:201-9. 